\title{
El proceso Bolonia y sus efectos en el campo de las Ciencias de la Actividad Física y del Deporte en España ${ }^{1}$
}

\author{
Ana Márcia Silva* \\ Susanna Soler** \\ Núria Puig*** \\ Maria Prat****
}

\begin{abstract}
Resumen: Este texto analiza el proceso de incorporación del sistema universitario español al Espacio Europeo de Educación Superior (EEES), más conocido como "proceso de Bolonia", centrándose en la implantación del grado en Ciencias de la Actividad Física y del Deporte (CAFyD). Para ello, se toma como referencia la teoría del campo científico de Bourdieu, desde cuya perspectiva se analiza el proceso acaecido. A partir del análisis de contenido de los documentos vinculados al EEES, y de entrevistas semiestructuradas a once personas claves del proceso, analizadas con apoyo del software NVivo 9.2®, los resultados indican dos aspectos especialmente relevantes: por una parte, una perspectiva positiva ante el proceso de Bolonia, si bien la distancia entre el modelo tradicional español y el nuevo modelo que se propone, así como la escasez de recursos, llevan a un modelo propiamente español y a la imposibilidad de aplicar las consignas del EEES, como sería deseable; y, por otra parte, la necesidad de las CAFyD por afirmarse en el sistema universitario, que lleva a iniciar el proceso con gran rapidez y de forma estricta, mediante la elaboración del Libro Blanco, por miedo a que el grado pudiera llegar a no ser reconocido.

Palabras clave: Educación Superior, Unión Europea, Educación Física. Deportes. Tratados.
\end{abstract}

\footnotetext{
${ }^{1}$ Ciencias de la Actividad Física y del Deporte es la nueva denominación del campo desde 1992, cuando dejó de llamarse Educación Física (EF) a partir del Real Decreto 1423.

*Faculdade de Educação Física. Universidade Federal de Goiás. Goiânia, GO. Brasil. E-mail: anamarcia@pq.cnpq.br

**Institut Nacional d'Educació Física de Catalunya, Barcelona, Espanha. E-mail: ssoler@ gencat.cat

***Institut Nacional d’Educació Física de Catalunya. Barcelona, Espanha. E-mail: npuig@ gencat.cat

****Universitat Autònoma de Barcelona. Barcelona, Espanha. E-mail: maria.prat@uab.cat
} 


\section{INTRODUCCIÓN}

Más que una joven frágil como Europa, ninfa de la mitología grecorromana cuyo nombre parece ser el origen de la denominación del continente (BACALARI, 2011), la Unión Europea (UE) ya es toda una dama. Con más de cincuenta años, la entonces llamada Comunidad Económica Europea (CEE) se ha constituido como un bloque político y económico desde su nacimiento en Roma en 1957. Aunque desde un punto de vista económico, la señora UE parece encontrarse en un momento delicado, desde una perspectiva cultural, educativa y científica, no quedan dudas de su gran solidez e influencia mundial.

También de nacimiento italiano, la Declaración de Bolonia, firmada en 1999 por los Ministros Europeos de Educación, desencadenó un proceso de fuertes cambios, entre los que destaca la creación del Espacio Europeo de Educación Superior (EEES). La firmaron 29 países, aunque apenas 24 de ellos estaban en la Eurozona, creada ese mismo año, y que implicaba la unión monetaria. En 2012, ya eran 47 los países adheridos al proceso Bolonia, formando parte del EEES e implementando las mismas políticas educativas en distintos contextos nacionales (EURYDICE, 2012). Como consecuencia, todas las instituciones de educación superior de los países firmantes deberían revisar sus carreras universitarias y adaptarlas a una nueva filosofía común.

En España, la Agencia Nacional de Evaluación de la Calidad y Acreditación (ANECA) estableció tres convocatorias consecutivas para que cada especialidad pudiera elaborar las nuevas directrices del ahora denominado título de Grado. En el ámbito específico, la Conferencia Española de Decanos de Facultades de CAFyD ${ }^{2}$ e Institutos Superiores de Educación Física se presentó en la segunda convocatoria para la elaboración del denominado Libro Blanco. Así, entre enero y junio de 2004 se elaboró el Libro Blanco de 1992, cuando dejó de llamarse Educación Física (EF) a partir del Real Decreto 1423.

Movimento, Porto Alegre, v. 20, n. 3, p. 1061-1082, jul./set. de 2014. 
CAFyD con la coordinación de la Facultad de Ciencias del Deporte de Extremadura.

El Libro Blanco fue la referencia para que los distintos centros de CAFyD elaboraran sus planes de estudio adaptados a las directrices de Bolonia. Entre ellos, el Institut Nacional d'Educació Física de Catalunya (INEFC), el segundo más antiguo del país con sus dos Centros adscritos a las universidades de Barcelona y de Lleida y funcionando respectivamente en estas dos ciudades, configuró su plan de estudios de Grado y Máster, entre 2007 y 2008. Por su experiencia histórica, diversidad en términos de vinculación universitaria, su visibilidad internacional y el importante papel político y académico que algunos de sus profesores han desempeñado en la escena nacional, este Instituto ha sido tomado como objeto de estudio para la investigación que se relata en el presente artículo.

El objetivo central de la investigación es analizar el sistema de relaciones entre instituciones y agentes que se estableció en el campo de las CAFyD y cómo éste influyó en la nueva configuración del Grado a partir de las directrices de Bolonia. Se analiza, pues, cómo éstas directrices fueron percibidas por los agentes del campo y como éstos las adaptaron a las circunstancias institucionales y a las relaciones establecidas entre los y las protagonistas del cambio.

Para llevar a cabo nuestro cometido hemos partido del concepto de campo científico propuesto por Bourdieu (1990, 1998, 2003, 2005, 2007). Este concepto es útil para entender el proceso permitiendo ir más allá de un relato de acontecimientos, ya que ayuda a comprender los enfrentamientos, las alianzas y el alcance de las medidas que se han desarrollado, dando sentido al proceso.

Según Bourdieu (2003), analizar un campo significa partir de un enfoque relacional, ya que estudia las interacciones que se establecen entre instituciones y agentes. Interacciones que tienen que ver con relaciones de fuerza y el ejercicio del poder, amistades y enemistades. Dado que la investigación llevada a cabo trata de la implantación de un nuevo modelo de Educación Superior, hay

Movimento, Porto Alegre, v. 20, n. 3, p. 1061-1082, jul./set. de 2014. 
que hablar de campo académico, universitario, en el que la ciencia ocupa una función cada vez más importante y, por tanto, es el eje en torno al cual se configura el campo. Un campo científico puede ser comprendido

[...] como sistema de las relaciones objetivas entre las posiciones adquiridas (en las luchas anteriores). Es el lugar (es decir, el espacio del juego) de una lucha de concurrencia, que tiene por apuesta específica el monopolio de la autoridad científica, inseparablemente definida como capacidad técnica y como poder social, o, si se prefiere, el monopolio de la competencia científica, entendida en el sentido de capacidad de hablar y de actuar legítimamente (es decir, de manera autorizada y con autoridad) en materia de ciencia. (BOURDIEU, 2005, p. 76)

En resumen, en nuestra investigación hemos analizado el campo científico de las CAFyD en el momento de la implantación del plan de Bolonia en tanto que una red de relaciones objetivas que actúa como un campo de fuerzas, cuyos límites están en juego con otros campos, más o menos consolidados, más o menos amplios, y en el cual juegan distintas instituciones y agentes, cada cual con sus capitales simbólicos específicos. Mediante las entrevistas a estos agentes nos ha sido posible conocer cuáles son estos capitales específicos y cómo han influido en el proceso. Enfatizamos en este texto como los documentos, en especial el Libro Blanco y consignas de Bolonia, se ajustan al entorno y son "vividos" por los protagonistas. En síntesis, la idea es que "el sistema de relaciones entre instituciones y agentes" es el que permite entender el punto actual, y por lo tanto, la subjetividad de los agentes es muy relevante.

Los resultados de este estudio, se estructuran en dos grandes apartados. En primer lugar, se presenta un análisis de cómo se ha llevado a cabo el proceso de Bolonia, con las peculiaridades de su desarrollo en España y sus límites y posibilidades de implementación. A continuación, se analiza cómo tuvo lugar este proceso específicamente en el campo de las CAFyD, planteando,

Movimento, Porto Alegre, v. 20, n. 3, p. 1061-1082, jul./set. de 2014. 
inicialmente, el papel estratégico que jugó el Libro Blanco en el campo y a continuación, se aborda el contexto de amenaza profesional en el mercado laboral y la necesidad de legitimación académica evidenciadas por los agentes del campo. En cada uno de los apartados, los documentos y las entrevistas realizadas "dialogan" con la teoría del campo científico, combinando análisis y esbozos de interpretación. Las conclusiones son una síntesis de los resultados obtenidos al tiempo que ponen de manifiesto el alcance y los límites de la investigación realizada.

\section{Metodología}

La metodología que se ha seleccionado para lograr el objetivo de esta investigación es de tipo cualitativo, combinando el análisis de contenido de documentos y el análisis de once entrevistas semiestructuradas, realizadas entre mayo y julio de 2012, identificados aquí con la letra "P" seguido del número de 1 a 11. Estas personas entrevistadas fueron seleccionadas intencionalmente en función de su contribución en el proceso de Bolonia y, en especial, en función de la posición clave que ocuparon en la elaboración del Libro Blanco de CAFyD, y en ANECA, incluyendo las aportaciones de personas que han tenido los cargos, entre otros, de la dirección de programas de ANECA, la dirección del INEFC, la dirección del centro de Barcelona de esta institución, así como de las personas responsables del proceso de creación y del proceso de implementación del nuevo plan de estudios y del cambio curricular. Las entrevistas fueron registradas previo consentimiento de las personas implicadas y se les garantizo el anonimato, incluso de sus cargos, pues quien conoce el caso podría identificar los informantes rompiendo el compromiso asumido. Posteriormente, las entrevistas fueron transcritas en su totalidad para poder realizar el análisis de contenido.

Por otra parte, los documentos analizados fueron los siguientes: el Real Decreto (RD) 1423/1992, la Declaración de Bolonia (Ministros Europeos de Educación, 1999), la Ley Orgánica

Movimento, Porto Alegre, v. 20, n. 3, p. 1061-1082, jul./set. de 2014. 
de Universidades (LOU) 06/2001; el Documento-Marco "La integración del sistema universitario español en el Espacio Europeo de Enseñanza Superior" del MECD (2003), el RD 1125/2003; el RD 55/2005; la Ley Orgánica 4/2007 por la que se modifica la LOU 6/2001; el RD 1892/2008 y el Libro Blanco (LB) de CAFyD.

El proceso de análisis de contenido se ha realizado con el apoyo del programa Nvivo $9^{\circledR}$, siguiendo los procedimientos habituales en esta técnica (BARDIN, 1996). Además, el programa permitió observar la mayor o menor similitud de términos usados en las diversas fuentes (documentales y entrevistas) y construir algunas categorías de análisis descritas en los apartados de este texto. La figura generada por este programa (Figura 1), muestra un mapa con las fuentes de datos agrupadas estadísticamente por similitud de palabras (análisis tipo cluster), mostrando, en primer lugar, el relativo distanciamiento y autonomía de percepción y reflexión ante el objeto de investigación de los diversos informantes. Por otra parte, la figura también nos indica que los documentos oficiales (RD y LB) están más lejos de la Declaración de Bolonia que las entrevistas a cada uno de los participantes del estudio (P). Esto, como veremos más adelante, confirma empíricamente la propuesta de interpretación teórica que hacemos en los apartados dedicados a los resultados.

Figura 1. Fuentes de información agrupadas por similitud de palabras

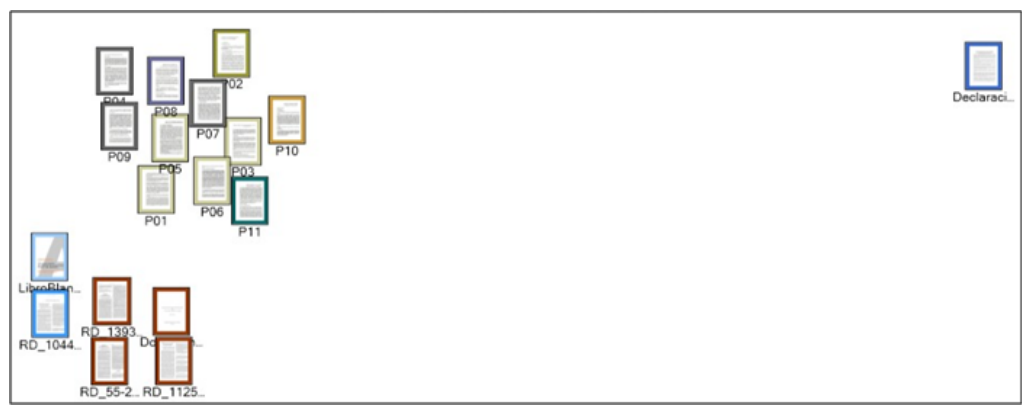

Fuente: Figura produzida pela própria equipe de pesquisa com o software Nvivo9@.

Movimento, Porto Alegre, v. 20, n. 3, p. 1061-1082, jul./set. de 2014. 


\section{UNA MIRADA AL PROCESO DE CONVERGENCIA DESDE LAS CAFYD}

La Declaración de Bolonia ha configurado un nuevo escenario en educación superior y sus rasgos más importantes son: la implantación generalizada del crédito europeo, denominado European Credit Transfer System (ECTS); currículos estructurados en dos ciclos (grado y postgrado) con un mínimo de 3 años para el primer ciclo; un sistema de titulaciones fácilmente comprensible y comparable que debe pasar por un proceso de acreditación para asegurar su calidad; y la transparencia mediante el Suplemento Europeo al Título (Ministros europeos de educación, 1999).

Es de destacar que no se ha obligado a los países a seguir el proceso de homogenización, sino que, tal y como se desprende de los documentos relativos al EEES el objetivo fue dar un incentivo al avance igualitario entre los países miembros de la UE, como exponía uno de los entrevistados: "Lo que Europa ha intentado en todo momento no ha sido obligar a los países, sino que a todos los países avanzaran de forma homogénea hacia esta idea de transparencia y de comparabilidad." (P11).

Para cumplir la agenda marcada por Europa, el gobierno español dio inicio al proceso mediante la LOU (2001). Su Título XIII está totalmente dirigido al entonces denominado Espacio Europeo de Enseñanza Superior, indicándose la importante responsabilidad del Consejo de Coordinación Universitaria en el proceso, y también anunciando la creación de ANECA, para coordinar los cambios, sobre todo en términos de acreditación y evaluación.

Como veremos, estas innovaciones parecen ser atractivas, pero las circunstancias del momento en que se han llevado a cabo han hecho muy difícil que alcanzaran sus objetivos.

Movimento, Porto Alegre, v. 20, n. 3, p. 1061-1082, jul./set. de 2014. 
3.1 EL DISEÑO DE LAS ENSEÑANZAS UNIVERSITARIAS EN EL MARCO DEL EEES: ¿ "UN SISTEMA DE BOLONIA A LA ESPAÑOLA"?

La estructuración del Grado en 4 años (240 ECTS) y del máster en 1 año (60 ECTS) en España, a diferencia de la gran mayoría de los países europeos, es uno de los temas que genera más divergencias en las entrevistas realizadas y en la documentación consultada, destacando al respecto la posición del MECD.

En el documento marco sobre la integración universitaria de España al EEES, se constata como el Ministerio sometió dos propuestas alternativas a consideración del Consejo de Coordinación Universitaria: en una se proponía un Grado de 240 ECTS (4 años), y en otra se proponía grados de 180 a 240 ECTS (entre 3 y 4 años), y 120 y 60 ECTS para los másteres (MECD, 2003). Finalmente, se optó por la primera propuesta, con grados de 240 ECTS y másteres de 60 ECTS (en una estructura de 4+1).

Con esta estructuración, el sistema universitario español ha resultado ser especialmente peculiar dentro del EEES, ya que de los 49 países que lo integraban en 2011, tan solo 6 tenían un sistema de grado organizado totalmente en 240 ECTS (Armenia, Chipre, Kazajistán, Georgia, Turquía y Ucrania). En los demás países que implementan el proceso de Bolonia prevalece el modelo de 180 créditos ECTS para el Grado y 120 para el Master (EURYDICE, 2012). Por este motivo, una de las personas entrevistadas no dudaba en afirmar: "Nosotros hemos hecho un sistema de Bolonia a la española" (P8).

En las entrevistas pudimos ver cómo surgieron distintas posiciones ante esta decisión. Para algunos, el $4+1$ no es un modelo acertado "Para mí ha sido un primer error que en España se haya tomado la decisión de 4+1. No lo entiendo, pero lo hemos de aceptar y trabajamos por el 4+1" (P1). Entre otros aspectos, se pone de relevancia que mantener 4 años de grado y 1 de máster hace mucho más difícil la armonización de planes de estudios con

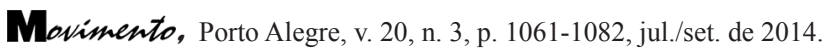


el resto de la UE, ya que la mayoría tienen establecida la fórmula de $3+2$.

Sin embargo, algunas voces plantean la importancia de respetar también la trayectoria histórica de cada país, de cada institución y de las especificidades de cada carrera: "nos tenemos que poner de acuerdo [...] pero, naturalmente, también hay que respetar la particularidad, la especificidad, el carácter y la personalidad de las instituciones, de las regiones y de los diferentes países de Europa y ahí es donde yo creo que está la cuestión, hasta donde estamos todos obligados a trabajar de la misma manera" (P4).

Algunas de las razones por las que se escogería la opción de un sistema 4+1 tendrían que ver con la tradición anterior de 5 años de licenciatura y las problemáticas internas que generaría un cambio de este tipo. Así, pasar a una formación de solo 3 años representaba reducir los programas y suprimir algunas materias, lo cual, en definitiva, ponía en peligro la estabilidad laboral del profesorado, como explica uno de los testimonios recogidos: "¿como iban a pasar de 5 años a 3? Había un miedo entre todos. Estrictamente es un tema laboral, un tema laboral y de conceptos, porque cada profesor cree que lo suyo es lo más importante" (P8).

El "miedo" al cambio es un elemento muy presente a la hora de valorar el proceso y los motivos de la decisión: Tenían miedo que se desmontara el sistema universitario español, de lo que pasaría con los profesores asociados que tenían; o sea, los que la crisis ahora ha echado fuera (P9).

Junto con las problemáticas laborales y políticas, otro testimonio también hace referencia a las implicaciones económicas, más que académicas, de la propuesta de $3+2$, ya que "En lugar de pagar un año de grado, se pagaría un año más de máster, y los ingresos son muy dispares" (P5). Las repercusiones económicas que implicaba el cambio, precisamente, fueron uno de los principales elementos de protesta ante la reforma universitaria durante el 2007 en todo el país por parte del alumnado y también de parte del profesorado.

Movimento, Porto Alegre, v. 20, n. 3, p. 1061-1082, jul./set. de 2014. 
En relación a la estructura del sistema universitario, otro de los debates presentes en el Estado español fue en torno a si el grado era suficiente para entrar en el mundo del trabajo o era preciso que, a continuación, el alumnado realizara el máster. En el Real Decreto $n^{\circ} 55 / 2005$ que establece la estructura de las enseñanzas universitarias, se considera que el grado es terminal; es decir, que habilita a la persona que lo obtenga a actuar en el mundo laboral. La propia Declaración de Bolonia indica literalmente que "el diploma obtenido después del primer ciclo será también considerado en el mercado laboral europeo como nivel adecuado de cualificación" (UE, 1999, p. 02).

Sin embargo, una de las críticas al nuevo modelo es también el carácter excesivamente generalista de las formaciones de Grado, de modo que el papel de los másteres toma relevancia: "El recorrido académico de cualquier estudiante debería ser de cinco años: grado más algo más para salir al mercado laboral" (P9). No obstante, el diseño de los másteres, especialmente en sus primeros momentos, en los que se crearon las primeras titulaciones de máster bajo el EEES sin haber diseñado aún las titulaciones de grado, también es objeto de crítica de los entrevistados.

En suma, se puede ver que había una distancia entre la propuesta de Bolonia y la realidad del sistema universitario dando lugar, tal como ya se ha apuntado, a un sistema de Bolonia "a la española”. En el siguiente apartado se describe cómo se implementó este proceso.

3.1.1 Sobre el Proceso de Implementación: Bolonia es "como UN CARAMELO QUE TE PONEN EN LA BOCA Y TE QUITAN ENSEGUIDA"

El proyecto Tuning Educational Structures in Europe (GONZÁLEZ; WAGENAAR, 2003; 2005), es clave para el proceso de convergencia en el ámbito metodológico. Asumido como documento oficial por el EEES, el proyecto Tuning propone una transformación de la cultura universitaria y la implantación de una nueva didáctica: el proceso pedagógico centrado en el aprendizaje

Movimento, Porto Alegre, v. 20, n. 3, p. 1061-1082, jul./set. de 2014. 
de los estudiantes y orientado a la adquisición de competencias, la evaluación continuada, y políticas de evaluación institucional y de acreditación del profesorado, como aspectos más relevantes.

Este nuevo enfoque pedagógico, así como la convergencia europea, se perciben como aspectos especialmente importantes y necesarios de forma prácticamente unánime por parte de todos los protagonistas de este estudio. Sin embargo, las posibilidades reales de implementar Bolonia generan una crítica un poco "ácida" entre los entrevistados, sobre todo por la escasez de recursos para desarrollar a la práctica un modelo basado, teóricamente, en la calidad de la enseñanza. Los datos oficiales de la UE confirman la tendencia al descenso de los presupuestos en educación superior en España, de modo que en el bienio 2009-2010 se registró una disminución del 2,9\% en relación al bienio anterior (EURYDICE, 2012, p.3).

Una de las personas entrevistadas comentaba: "Esto hay que pagarlo, pero luego no hay dinero. Además, en un contexto de crisis como el que estamos actualmente [...], es que te ponen el caramelo en la boca, y después te lo quitan enseguida." (P2). Otra decía: "El plan de Bolonia es como un Ferrari" (P5). Los indicios muestran que Bolonia es, efectivamente, un plan educativo tan potente como un Ferrari, pero el mismo testimonio nos planteaba que "son tiempos duros para mantener un Ferrari". A continuación, se muestran algunos ejemplos de esta contradicción entre las directrices existentes (la teoría), y el contexto en que se han tenido que implantar (la práctica), a partir de la LOU de 2001.

Algunos efectos concretos de las limitaciones económicas se observan en la rigidez y el encorsetamiento de la oferta de créditos y la homogeneización curricular. Con el paso de la licenciatura a grado, en el caso específico de CAFyD en el INEFC, se hace patente la pérdida de optatividad y la delimitación del formato de las asignaturas y la configuración de grupos. También se observan en la reducción de profesorado, que conlleva la configuración de grupos con muchos alumnos en clase, dificultando, o incluso

Movimento, Porto Alegre, v. 20, n. 3, p. 1061-1082, jul./set. de 2014. 
imposibilitando, llevar a la práctica el modelo teórico, hecho que repercute en la calidad de la enseñanza.

Junto con la reducción de recursos, algunas voces también destacan la falta de motivación de una parte del profesorado para implementar los cambios, así como la insuficiente formación recibida a lo largo del proceso para poder introducir el nuevo enfoque pedagógico.

Finalmente, Bolonia también ha traído nuevas exigencias al profesorado en relación a los procesos de acreditación por parte de agencias externas creadas a tal fin. Este aspecto recibe también una gran cantidad de críticas, sobre todo por la priorización de la tarea investigadora y la publicación científica, por encima de la tarea docente y divulgativa, en los criterios de evaluación establecidos por estas agencias.

Así, además de las dificultades económicas, se pone de manifiesto que la nueva propuesta significa otra forma de organización del sistema universitario, generando una gran ruptura, ya que va en contra de uno de los ejes identificadores que es la docencia en el campo de CAFyD.

Por ello, se puede afirmar que "el ingreso en el campo supone una adhesión paradójica a un conjunto más o menos radicalmente nuevo de presupuestos $\mathrm{y}$, de modo correlativo, el descubrimiento de apuestas y exigencias desconocidas e incomprendidas por la experiencia habitual" (BOURDIEU, 1998, p. 24).

Ahora bien, si en el apartado que ahora finaliza hablamos de contradicciones y resistencias dentro del campo, en los siguientes veremos algunos aspectos que, paradójicamente, hacen que, precisamente el ámbito de las CAFyD haya sido uno de los más dinámicos a la hora de implantar Bolonia en sus planes de estudios. 


\section{El proceso de Bolonia en Cafyd: los retos que debe AFRONTAR UN CAMPO CIENTÍFICO NUEVO ANTE EL CAMBIO}

\subsection{EL LIBRO BLANCO COMO "UNA BRÚJULA" Y SUS OTROS EFECTOS EN EL CAMPO}

La metáfora que forma parte del título de este ítem habla de la función y también de la fuerza política que ha obtenido el LB en los procesos de configuración de los planes de estudio en España. Su importancia es inequívoca en el proceso histórico de la titulación en CAFyD, y su papel orientativo se pone en evidencia en las palabras de uno de los entrevistados: "lo seguimos como la estrella polar, la brújula iba hacia el LB” (P9).

Tal y como afirma quien fue un importante cargo en ANECA en los inicios del proceso, los estudios de CAFyD estuvieron en las primeras convocatorias de elaboración del LB a instancias suyas: "Como coordinador de la elaboración del LB de todas las titulaciones, tengo que decirlo ahora, [el de CAFyD] fue uno de los que también propuse, para que se iniciara el proceso con la EF. [...] Fue un trabajo muy duro, en realidad una puesta en escena de lo que podrían ser, en el futuro, los estudios de EF." (P11). Mediante este testimonio, también se puede advertir como este autorizado agente, vinculado al sistema universitario español, concedía una valiosa importancia académica a la carrera - que aún llama de "Educación Física" -, para situarla en la Universidad.

La coordinación de su elaboración fue llevada a cabo por la Facultad de Ciencias del Deporte de la Universidad de Extremadura, y más concretamente, por su decano, que en aquel momento también era presidente de la Conferencia Española de Institutos y Facultades de Ciencias de la Actividad Física y del Deporte, así como de la Asociación Española de Ciencias del Deporte (AECD). Se trataba de una facultad muy joven $y$, sin duda, este es un factor muy importante para que asumiera esta función, ya que necesitaba afirmarse en el campo científico de las CAFyD, y también dentro de

Movimento, Porto Alegre, v. 20, n. 3, p. 1061-1082, jul./set. de 2014. 
su universidad. Bajo esta coordinación, participaron en el proceso los 22 centros que en ese momento impartían los estudios, hecho que fue muy bien valorado por la ANECA.

El resultado final del Libro Blanco, también refleja relaciones de fuerza en el campo, pues hay un predominio de contenidos relacionados con el deporte y la salud cuando, en realidad, las salidas profesionales de las CAFyD son principalmente la docencia y la gestión (VIÑAS, PUIG \& PÉREZ, 2009). Las sugerencias de Hernández y Rodríguez (2006) también perseguían ampliar el campo de trabajo de los futuros profesionales, pero apostaban por una distribución más equilibrada entre los distintos ámbitos de contenido.

La cultura hegemónica de los contenidos del deporte, así como de la salud, puede encontrar explicaciones en sus raíces históricas en España (PASCUAL BAÑOS, 1997; PASTOR PRADILLO, 2007). Uno de los entrevistados enfatiza que las competencias que propone el LB están distantes de las necesidades profesionales: "En el Libro Blanco están muy hinchadas todas las competencias de salud" (P10).

A pesar de esto, curiosamente, el LB Blanco indica que el Área de Conocimiento más adecuada para el campo de CAFyD es la de las Ciencias Sociales y Jurídicas, e incluso el proyecto de Libro Blanco en CAFyD fue inscrito en esta área en la segunda convocatoria de ANECA, siguiendo la vinculación tradicional al área más pedagógica y humanística. Según uno de los integrantes de la Conferencia en aquel momento, nadie planteó el Área de la Salud como prioritaria, apareciendo solamente como secundaria, si bien esta posibilidad existía por el carácter generalista que tenía la propuesta.

En definitiva, se pone en evidencia que el resultado final no fue fruto de un análisis objetivo de las necesidades en el mercado de trabajo, sino de las relaciones de fuerza en el campo y, en este caso, de la mayor fuerza que ejercieron las personas vinculadas a las disciplinas relacionadas con el deporte y la salud. La 
divergencia en los puntos de vista, se constata claramente en los resultados finales de la votación sobre el LB, ya que más de un cuarto de los representantes no aprobó el documento tal y como estaba desarrollado (ANECA, 2004).

\subsection{EL GRADO EN CAFYD: ¿“BAJO EL PRISMA DEL MIEDO”?}

A finales del siclo XX comenzó a tener lugar la incorporación a las universidades de los centros que impartían los estudios de CAFyD y se crearon las facultades correspondientes. En el 2013 todos los antiguos y nuevos centros, ya son facultades o forman parte de facultades, a excepción del INEF de Cataluña, el cuál a pesar de ser organismo autónomo, igualmente se rige por la misma normativa universitaria que el resto. Esta incorporación no ha estado exenta de dificultades: "Ha sido una crisis lo que ha supuesto estar en el ámbito universitario.” (P3)

Al ser un campo nuevo y en proceso de consolidación, la adaptación de los planes de estudio a Bolonia significó un nuevo reto de cara a la legitimación de campos todavía muy jóvenes en el panorama universitario español. Así se comprende que la elaboración del LB, cumpliendo el cronograma y las indicaciones de ANECA, así como las configuraciones de todos los planes de estudio: "Las CAFyD es la materia, posiblemente de todas las materias de todo el Estado, que cumplen más al pie de la letra el Libro Blanco, porque creo que los planes de estudio de CAFyD se hicieron bajo la sombra del miedo." (P10)

Los campos académicos con gran reconocimiento social y político como Medicina, Ingeniería o Derecho, no tenían miedo, pero esta situación todavía no existía en el campo de las CAFyD, así que urgía cumplir muy rigurosamente las nuevas pautas para tener el menor riesgo posible a los ya existentes. La desaparición de la especialidad de Magisterio de Educación Física (MEF), quedando diseminada dentro de la formación de maestros generalistas de primaria, incrementaba más aún este temor. 
Por otra parte, la urgencia por realizar el LB tan rápidamente, también se explica para no dejar que otros campos profesionales colonizasen la especificidad del campo de las CAFyD: "es marcar territorio, es marcar territorio. Esto es la explicación de porqué al principio, porque de forma rápida [...]" (P8). Otro protagonista también hace referencia a las "fronteras" de las CAFyD y por donde se percibe la amenaza profesional: "Por una parte, hay una formación de maestros que tienen un ámbito específico, y por otra, también están todos los aspectos de salud, que a veces están en la frontera dentro de los estudios de CAFyD" (P11).

Los protagonistas del LB tenían en sus manos la gestión de todas las instituciones que impartían la formación en CAFyD, estaban involucrados en el sentido del juego del campo científico. Su responsabilidad en tomar decisiones y prever los efectos sobre el campo, provenían de su biografía personal, pero especialmente de su inmersión en el proceso que les constituyó como académicos. Como decía Bourdieu, estos sujetos

[...] han incorporado un sinfín de esquemas prácticos de percepción y de valoración que funcionan en tanto que instrumentos de construcción de la realidad, en tanto que principios de visión y de división del universo en el que se mueven, no necesitan plantear como fines los objetivos de su práctica. No son como sujetos frente a un objeto (o, menos aún, frente a un problema) que estaría constituido como tal por un acto intelectual de conocimiento; están, como se dice, metidos de lleno en el quehacer (BOURDIEU, 2007, p. 145).

Los datos indican que el proceso de Bolonia y sus efectos en las CAFyD, con la elaboración del Libro Blanco, representaran momentos importantes de crisis de un campo aún en consolidación, con una apuesta por conseguir reconocimiento social. 


\section{ConsidERACIONES FINALES}

El análisis del proceso de implantación del plan de Bolonia a los estudios de CAFyD en España desde la perspectiva teórica del campo científico propuesta por Bourdieu permite entenderlo en toda su complejidad y ayuda a huir de la simple anécdota. Este autor nos indica la necesidad de conocer científicamente las condiciones sociales por medio de las cuales ocurre la construcción científica, y los sujetos de estas construcciones, además de los propios productos y políticas científicas (BOURDIEU, 2003).

En nuestro trabajo hemos puesto de manifiesto tres cuestiones fundamentales: 1. La gran distancia entre el punto de partida (el sistema universitario español) y el objetivo final (el modelo y las pautas del EEES), materializado en una concepción muy diferente de los estudios universitarios; 2. El acuerdo unánime por parte de todos los agentes del campo de las CAFyD por adaptarse rápidamente a Bolonia y el liderazgo asumido por uno de ellos; y 3. La búsqueda de una legitimidad del campo motivada por el miedo a no ser reconocido o colonizado por otros campos profesionales más consolidados.

Del análisis de las entrevistas destaca la fuerza de los agentes responsables del proceso, así como también la conducción activa de los demás al juzgar las fuerzas sociales, económicas, políticas y académicas de aquel contexto. Esta comprensión de los agentes indica la incorporación del sentido del juego científico que constituye el habitus científico como identifica Bourdieu (1998) implicando en esquemas de percepción y de acción, de conocimiento y reconocimiento de las leyes del juego científico, así como también de lo que está en juego cuando se juega.

Finalmente, el miedo a que los estudios de grado en CAFyD pudieran desaparecer y a la intromisión de otros campos científicos más consolidados, ha sido otro de los "motores" del campo en la búsqueda de su legitimidad. Había que trabajar mejor y más rápido que nadie en la construcción del LB, así como después con los

Movimento, Porto Alegre, v. 20, n. 3, p. 1061-1082, jul./set. de 2014. 
planes de estudio para afirmarse en el espacio de la educación superior, reaccionando ante las amenazas.

En este artículo hemos querido ofrecer algunos elementos para comprender la implantación de Bolonia en las CAFyD desde la perspectiva de la teoría del campo científico, habiendo desvelado el sistema de relaciones entre instituciones y agentes que se estableció frente a la nueva configuración del Grado. Se recomiendan nuevos estudios que abarquen una gama más amplia de entrevistados, incluyendo especialmente al alumnado universitario que, sin estar en el día a día de la elaboración de los planes de estudio, manifestó su parecer en otros foros que no se han tenido en cuenta en este artículo- y también la perspectiva de una facultad, vinculada orgánicamente a una Universidad, lo que posibilitaría una comparación, contraste y ampliación de la investigación aquí relatada.

Aprender lo que se ha hecho en esta primera década del siglo y también de lo que no se ha hecho en los estudios universitarios, y sobre todo en CAFyD, es una nueva lección de historia que nos ayudará a organizarnos para un futuro mejor. 
O processo Bolonha e seus efeitos no campo das ciências da atividade física e do esporte em Espanha

Resumo: Este texto analisa o processo de incorporação do sistema universitário espanhol ao Espaço Europeu de Educação Superior (EEES), mais conhecido como "processo Bolonha", enfocando a implantação do grau em Ciências da Atividade Física e do Desporto (CAFyD). Para isso, toma-se como referência a teoria de campo científico de Bourdieu, de cuja perspectiva analisa-se o processo em foco. A partir da analise de conteúdo dos documentos vinculados ao EEES e de entrevistas semi-estruturadas a onze pessoas chaves do processo, analisados com apoio do software NVivo $9.2 \AA$, os resultados indicam dois aspectos especialmente relevantes: por uma parte, uma perspectiva positiva frete ao processo de Bolonha, se bem que a distância entre o modelo tradicional espanhol e o novo modelo que se propõe, assim como a escassez de recursos, resultam em um modelo propriamente espanhol e a uma impossibilidade de aplicar os princípios do EEES como seria desejável; e por outra parte, a necessidade das CAFyD de afirmar-se no sistema universitário induz iniciar o processo com grande rapidez e de forma objetiva mediante a elaboração do LB, por medo de que esta graduação pudesse não ser reconhecida.

Palavras-chave: Educação Superior. União Europeia. Educação Física. Esportes. Tratados.

The Bologna process and its impact on the field of physical activity and sport sciences in Spain

Abstract: This paper examines the process of incorporation of the Spanish university system to the European Higher Education Area (EHEA), known as "Bologna process". We focus on implementation of the Degree on Physical Activity and Sport Sciences (PASS). We examine that process from Bourdieu's theory of scientific field. Data obtained through content analysis of documents related to the EHEA and semi-structured interviews with eleven key actors in the process were analyzed with the support of NVivo $9.2 ®$ software. Results indicate two particularly relevant aspects. Firstly, a positive attitude towards the Bologna process, even though the distance between the Spanish traditional model and the new one, as well the scarcity of resources, result in an actual Spanish model and hamper the application of EHEA principles as desirable. Secondly, the PASS's need to assert themselves within the university system leads this discipline to start the process very quickly and strictly through the development of the White Paper, out of the fear that the university degree would not be recognized.

Keywords: Higher Education. European Union, Physical Education. Sports. Treaties. 


\section{REFERENCIAS}

AGENCIA NACIONAL DE EVALUACIÓN DE LA CALIDAD Y ACREDITACIÓN. Libro Blanco. Título de Grado en Ciencias de la Actividad Física y del Deporte. Madrid, 2004. Disponible en: <http://www.aneca.es/var/media/150296/libroblanco_ deporte_def.pdf>. Acceso en: 21 abr. 2011.

BACALARI, A. EI mito de Europa en los textos literarios clásicos. Acta Literaria. Concepción, n. 43, p. 95-109, 2011.

BARDIN, L. Análisis de contenido. 2.ed.. Madrid: Akal, 1996.

BOURDIEU, P. Sociología y cultura. México: Grijalbo, 1990.

BOURDIEU, P. Meditaciones Pascalianas. Barcelona: Anagrama, 1998.

BOURDIEU, P. Ciencia de la ciencia y reflexividad. Barcelona: Anagrama, 2003.

BOURDIEU, P. Intelectuales, política y poder. Buenos Aires: EUDEBA, 2005.

BOURDIEU, P. Razones prácticas. Sobre la teoría de la acción. Barcelona. Anagrama, 2007.

EUROPEAN NETWORK OF SPORT SCIENCE, EDUCATION \& EMPLOYMENT. Proyecto de Red Temática AEHESIS: Aligning a European Higher Education Structure In Sport Science, 2006. Disponible en: <http://www.aehesis.de/images/ FilesForDL/Summaries/SpanishSummary.pdf>. Acceso en: 21 abr. 2011.

EURYDICE (Education, Audiovisual and Culture Executive Agency). The European Higher Education Area in 2012: Bologna Process Implementation Report. Brussels/Belgium, 2012. Disponible en: <http://eacea.ec.europa.eu/education/ eurydice>. Acceso en: 26 sep. 2012.

GONZÁLEZ, J.; WAGENAAR, R. G. (eds.). Tuning Educational Structures in Europe. Final Report, Phase One. Bilbao: Groningen, Universidad de Deusto, University of Groningen, 2003.

GONZÁLEZ, J., WAGENAAR, R. G. (eds.). Tuning Educational Structures in Europe. Final Report. Bilbao: Groningen, Universidad de Deusto, University of Groningen, 2005.

HERNÁNDEZ, J., RODRÍGUEZ, J. P. Los contenidos exclusivos de la formación de los docentes de Educación Física: el camino hacia la convergencia europea. Revista Iberoamericana de Educación, Madrid, Buenos Aires, n. 40, v. 6, dic. 2006. Disponible en: <http://www.rieoei.org/deloslectores/1588Hernandez.pdf>. Acceso en: 28 feb. 2012.

LEY Orgánica 6/2001, de 21 de diciembre, de Universidades. Boletín Oficial del Estado, n. 307, de 24 diciembre 2001, p. 49400- 49425.

LEY Orgánica 4/2007, de 12 de abril, por la que se modifica la Ley Orgánica 6/2001,

Movimento, Porto Alegre, v. 20, n. 3, p. 1061-1082, jul./set. de 2014. 
El proceso bolonia y sus efectos en el campo de las ciencias ...

de 21 de diciembre, de Universidades. Boletín Oficial del Estado, n. 89, de 13 de abril de 2007, p. 16241- 16260.

MINISTERIO DE EDUCACIÓN, CULTURA Y DEPORTE. La integración del sistema universitario español en el Espacio Europeo de Enseñanza Superior: Documento-marco, 2003. Disponible en: <http://www.uma.es/publicadores/eees/ wwwuma/documentomarco.doc>. Acceso en: 25 abr. 2012.

MINISTROS EUROPEOS DE EDUCACIÓN. Declaración conjunta de los Ministros Europeos de Educación reunidos en Bolonia el 19 de Junio de 1999. Disponible en: <http://www.universia.es/contenidos/universidades/documentos/ Universidades_docum_bolonia.htm>. Acceso en: 21 oct. 2010.

PASCUAL BAÑOS, C. Análisis contextual en la formación del profesorado de educación física. Revista de Educación, Madrid, n. 313, p. 161-178, 1997.

PASTOR PRADILLO, J. L. De la identidad profesional y la ocasión histórica. Revista de Comunicación Intercolegial. Madrid, n. 2, p. 25, jul./feb. 2007.

REAL Decreto 1423/1992, de 27 de noviembre, sobre la incorporación a la universidade de las enseñanzas de Educación Física. Boletín Oficial del Estado, n. 307, de 23 diciembre 1992, p. 43641-43643.

REAL Decreto 1125/2003, de 5 de septiembre, por el que se establece el sistema europeo de créditos y el sistema de calificaciones de las titulaciones universitarias de carácter oficial y validez en todo el territorio nacional. Boletín Oficial del Estado, n. 224, de 18 septiembre 2003, p.34355-34356.

REAL Decreto 55/2005, de 21 de enero, por el que se establece la estructura de las enseñanzas universitarias y que se regulan los estudios universitarios oficiales de grado. Boletín Oficial del Estado, n. 21, de 25 enero 2005, p. 2842-2846.

REAL Decreto 1892/2008, de 14 de noviembre, por el que se regulan las condiciones para el acceso a las enseñanzas universitarias oficiales de grado y los procedimientos de admisión a las universidades públicas españolas. Boletín Oficial del Estado, n. 283, de 24 noviembre 2008, p. 46932-46946.

VIÑAS, J.; PUIG, N.: PÉREZ, M. El mercat de treball en l'esport. Llicenciatura de I'INEFC-Barcelona. Barcelona: Diputació de Barcelona, 2009.

Financiamento: CAPES (Coordenadoria de Aperfeiçoamento de Pessoal de Nível Superior).

Movimento, Porto Alegre, v. 20, n. 3, p. 1061-1082, jul./set. de 2014. 
Endereço para correspondência:

Faculdade de Educação Física

Campus Samambaia

Caixa Postal 131

CEP: 74001-970 Goiânia - Goiás

Recebido em: 08.10.2013

Aprovado em: 30.03.2014

Movimento, Porto Alegre, v. 20, n. 3, p. 1061-1082, jul./set. de 2014. 